\title{
lifelines: survival analysis in Python
}

\section{Cameron Davidson-Pilon ${ }^{1}$}

\section{DOI: $10.21105 /$ joss. 01317}

\section{Software}

- Review ¿

- Repository ca

- Archive ${ }^{\top}$

Submitted: 06 March 2019 Published: 04 August 2019

\section{License}

Authors of papers retain copyright and release the work under a Creative Commons Attribution 4.0 International License (CC-BY).
1 Independent researcher

\section{Summary}

One frustration of data scientists and statisticians is moving between programming languages to complete projects. The most common two are $\mathrm{R}$ and Python. For example, a survival analysis model may be fit using R's survival-package (Terry M Therneau, 2015) or flexsurv (Christopher Jackson, 2016), but needs to be deployed into a Python system. Previously, this may have meant using Python libraries to call out to $\mathrm{R}$ (still shuffling between two languages, but now abstracted), or translating the fitted model to Python (likely to introduce bugs). Libraries like Patsy (Nathaniel J. Smith et al., 2018) and Statsmodels (Skipper Seabold et al., 2017) have helped data scientists and statisticians work in solely in Python. lifelines extends the toolbox of data scientists so they can perform common survival analysis tasks in Python. Its value comes from its intuitive and well documented API, its flexibility in modeling novel hazard functions, and its easy deployment in production systems \& research stations along side other Python libraries. The internals of lifelines uses some novel approaches to survival analysis algorithms like automatic differentiation and meta-algorithms. We present high-level descriptions of these novel approaches next.

One goal of lifelines is to be pure Python so as to make installation and maintenance simple. This can be at odds with users' desire for high-performance model fitting. Though Python is becoming more and more performant, datasets are getting larger and larger at a faster rate. Internally, lifelines uses some interesting tricks to improve performance. These approaches can be applied to other Python libraries. For example, the Cox proportional hazard model with Efron's tie-handling method has a complicated partial-likelihood (Wikipedia contributors", 2019):

$$
\ell(\beta)=\sum_{j}\left(\sum_{i \in H_{j}} X_{i} \cdot \beta-\sum_{\ell=0}^{m-1} \log \left(\sum_{i: Y_{i} \geq t_{j}} \theta_{i}-\frac{\ell}{m} \sum_{i \in H_{j}} \theta_{i}\right)\right)
$$

where $\theta_{i}=\exp \left(X_{i} \cdot \beta\right)$, and the Hessian matrix is:

$$
\ell^{\prime \prime}(\beta)=-\sum_{j} \sum_{\ell=0}^{m-1}\left(\frac{\sum_{i: Y_{i} \geq t_{j}} \theta_{i} X_{i} X_{i}^{\prime}-\frac{\ell}{m} \sum_{i \in H_{j}} \theta_{i} X_{i} X_{i}^{\prime}}{\phi_{j, \ell, m}}-\frac{Z_{j, \ell, m} Z_{j, \ell, m}^{\prime}}{\phi_{j, \ell, m}^{2}}\right)
$$

where

$$
\begin{gathered}
\phi_{j, \ell, m}=\sum_{i: Y_{i} \geq t_{j}} \theta_{i}-\frac{\ell}{m} \sum_{i \in H_{j}} \theta_{i} \\
Z_{j, \ell, m}=\sum_{i: Y_{i} \geq t_{j}} \theta_{i} X_{i}-\frac{\ell}{m} \sum_{i \in H_{j}} \theta_{i} X_{i} .
\end{gathered}
$$


These could be implemented in Python using, for example, Python's native for loops. However, this would be too slow. We would like to use NumPy, which offers vectorized operations. However, even with NumPy, there are still some Python for loops. lifelines implements these equations using NumPy's einsum function, which is used to express tensor products in Einstein summation notation. The result is that the tensor products, which are just for loops, are pushed down to as close to the $C$ layer as possible. From internal tests, using einsum resulted in a $4 x$ speed improvement.

Another optimization in the lifelines' implementation of Cox proportional hazard model is using a meta-algorithm to pick the most performant algorithm at runtime. There are two algorithms that can be used to compute the partial likelihood (and its gradient and Hessian). One algorithm is faster when there is a high cardinality of times in the dataset (low count of ties), and the other is faster when there low cardinality of times (high count of ties). There is not a simple heuristic of when one is faster than the other, at it depends on other factors like the size of the dataset and the average count of data points per time. To overcome this, I generated hundreds of artificial datasets of varying size, varying cardinality of times, and varying average count of data points per time. After running both algorithms against the datasets and recording their durations, I fitted a linear regression model to predict the ratio of duration. This model is accurate (R-squared over $80 \%$ ), easy to implement, and importantly can predict very fast. Thus, at runtime, we compute summary values for the dataset and let the linear model predict which algorithm will be faster. By choosing the appropriate algorithm, we achieve an up-to $3 x$ performance improvement on some datasets. When other performance improvements are made to either algorithm, we rerun the dataset generation and model training to get a new linear model for prediction.

A non-performance innovation in lifelines is the incredible flexibility for user-defined parametric cumulative hazards. This allows the user to specify precise parametric models and perform inference and statistical tests on them. This generalization of parametric models, which previously was confined to well-known probability distributions, is possible due to the automatic differentiation engine, Autograd, and the fact that any probability distribution can be defined by a cumulative hazard function. lifelines has implemented the log-likelihood for an arbitrary cumulative hazard, $H(t \mid \theta)$, including possible censoring and left-truncation:

$$
\log l(\theta \mid t, c, s)=\sum_{i: c_{i}=1}\left(\log h\left(t_{i}\right)-H\left(t_{i}\right)\right)-\sum_{i: c_{i}=0} H\left(t_{i}\right)+\sum_{i} H\left(s_{i}\right)
$$

where $t_{i}$ are the observed or censored times of subject $i, c_{i}$ is 0 if the subject was censored, $s_{i}$ is time the subject entered the study, and $h(t)$ is the hazard which is the derivative of the cumulative hazard with respect to time. We use automatic differentiation to compute $h(t)$ and the gradient and Hessian with respect to the unknown parameters $\theta$. The user only needs to specify a cumulative hazard (in Python code). lifelines will invoke SciPy's minimize with the computed derivatives and return the maximum likelihood estimators of the model, along with standard errors, p-values, confidence intervals, etc. An example user defined cumulative hazard is below:

from lifelines.fitters import ParametericUnivariateFitter

class ThreeParamHazardFitter(ParametericUnivariateFitter):

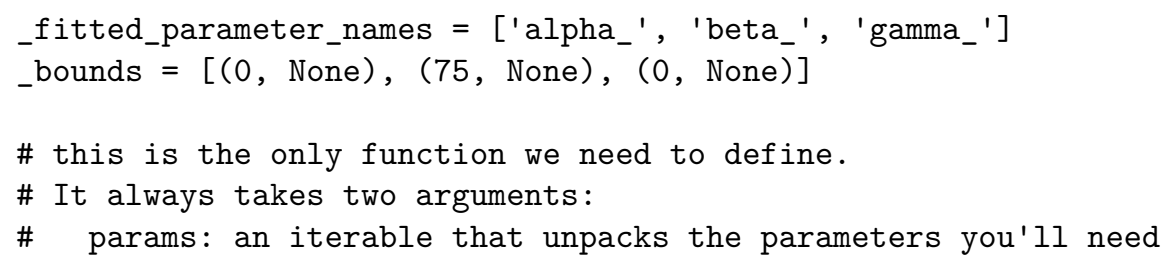




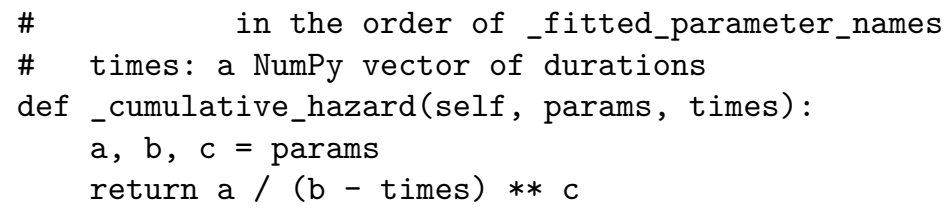

Some more examples of user-defined cumulative hazards are in the main documentation, including how to add covariates.

\section{Acknowledgments}

l'd like to acknowledge all the researchers in survival analysis, specifically Terry Therneau and the other authors of R's survival package. I'd also like to acknowledge the contributers to the lifelines projects.

\section{References}

Christopher Jackson. (2016). flexsurv: A platform for parametric survival modeling in R. Journal of Statistical Software, 70(8), 1-33. doi:10.18637/jss.v070.i08

Nathaniel J. Smith et al. (2018, October). Pydata/patsy: V0.5.1. doi:10.5281/zenodo. 1472929

Skipper Seabold et al. (2017, February). Statsmodels/statsmodels: Version 0.8 .0 release. doi:10.5281/zenodo. 275519

Terry M Therneau. (2015). A package for survival analysis in s. Retrieved from https: //CRAN.R-project.org/package=survival

Wikipedia contributors". (2019). Proportional hazards model - Wikipedia, the free encyclopedia. Retrieved from https://en.wikipedia.org/w/index.php?title=Proportional_hazards_ model\&oldid=885553852 